\title{
Science of Synthesis: Catalytic Transformations via C-H Activation, Workbench Edition, 2 Vol.
}

\author{
Edited by Jin-Quan Yu, $1^{\text {st }}$ ed., Thieme: Stuttgart, 2016, 1044 pp, € 449, ISBN: 9783132400511
}

Methods for the direct functionalization of otherwise inert carbon-hydrogen $(\mathrm{C}-\mathrm{H})$ bonds have emerged as a transformative platform in molecular synthesis. While early contributions had focused on stoichiometric transformations, recent years have witnessed the development of various protocols for catalytic $\mathrm{C}-\mathrm{H}$ transformations. This strategy has found increasingly viable applications in diverse research areas, ranging from material sciences to natural product synthesis and pharmaceutical industries. The two Science of Synthesis volumes entitled Catalytic Transformations via $\mathrm{C}-\mathrm{H}$ Activation edited by Prof. Jin-Quan Yu provide a comprehensive summary of all relevant aspects of $\mathrm{C}-\mathrm{H}$ functionalizations, covering among others organometallic $\mathrm{C}-\mathrm{H}$ activation transformations, carbene- and nitrene-mediated reactions as well as radical-based functionalizations.

The contributions are divided into two volumes, with the first one addressing $\mathrm{C}\left(\mathrm{sp}^{2}\right)-\mathrm{H}$ functionalizations for $\mathrm{C}-\mathrm{C}$ formatting processes. Thus, the first chapters provide a thorough overview of transition-metal-catalyzed arene arylations, alkenylations and alkylations by the use of broadly applicable transition-metal complexes, mostly focusing on palladium and ruthenium catalysis manifolds. This volume is complemented by an excellent chapter on step-economical C-C activations of heteroarenes, highlighting the versatility of this approach. The second volume summarizes the state-ofthe-art in $\mathrm{C}-\mathrm{H}$ transformations by carbene and nitrene intermediates as well as transformations by radical intermediates. These outstanding reviews are accompanied by chapters on $\mathrm{C}-\mathrm{H}$ activations for oxidative transformations and carbon-heteroatom formations, featuring the remarkable advances in catalyzed direct halogenations, oxygenations, nitrogenations and borylations.
All chapters are written by internationally leading experts in a clear and reader-friendly manner. The individual contributions are characterized by highly useful experimental sections, illustrating typical procedures for synthetically meaningful C-H functionalizations.

$\mathrm{C}-\mathrm{H}$ activation chemistry is a rapidly evolving research arena. Since the majority of references cover research until 2013, scientists that are interested in very recent developments of $\mathrm{C}-\mathrm{H}$ functionalization will likely consult further topical reviews on the specific aspects of interest. While a variety of reviews on $\mathrm{C}-\mathrm{H}$ activation chemistry were published during the past decade, the two Science of Synthesis volumes represent a comprehensive overview on the topic, which should prove invaluable for scientists in academia as well as the practitioners in applied areas, such as chemical, agrochemical and pharmaceutical industries. Particularly, the experimental sections of the individual chapters should prove instrumental for the reader to evaluate and apply the $\mathrm{C}-\mathrm{H}$ functionalization protocols. Overall, I strongly recommend these two volumes on Catalytic Transformations by $\mathrm{C}-\mathrm{H}$ Activation as an excellent reference collection for all academic and industrial researchers being interested in $\mathrm{C}-\mathrm{H}$ activation. In consideration of the increasing practical importance of applied $\mathrm{C}-\mathrm{H}$ functionalizations, these two volumes are expected to stimulate exciting new trends in $\mathrm{C}-\mathrm{H}$ activation catalysis.

Lutz Ackermann, Georg-August-Universität, Göttingen, Germany 